\title{
SOME THEOREMS ON FOURIER COEFFICIENTS
}

WALTER RUDIN ${ }^{1}$

I. Trigonometric polynomials with coefficients \pm 1 . Consider the trigonometric polynomial

$$
P\left(e^{i \theta}\right)=\sum_{n=1}^{N} \epsilon_{n} e^{i n \theta}
$$

where $\epsilon_{n}= \pm 1$. If we set $\|P\|_{\infty}=\max _{\theta}\left|P\left(e^{i \theta}\right)\right|$, the Parseval theorem shows that $\|P\|_{\infty} \geqq N^{1 / 2}$, and the following problem arises: does there exist an absolute constant $A$ with the property that for each $N$ one can find $\epsilon_{1}, \cdots, \epsilon_{N}$, equal to \pm 1 , so that

$$
\|P\|_{\infty} \leqq A N^{1 / 2}
$$

where $P$ is given by (1.1)?

If one allows the coefficients $\epsilon_{n}$ to be complex numbers of absolute value 1 , an affirmative answer to the question is furnished by the partial sums of the series $\sum_{1}^{\infty} e^{i n \log n} e^{i n \theta}$; this example is due to Hardy and Littlewood [4,pp. 116-118]. A theorem of Salem and Zygmund $\left[2\right.$, pp. 270, 278] shows, roughly speaking, that $(N \log N)^{1 / 2}$ is the "most probable" order of magnitude for $\|P\|_{\infty}$ if $\epsilon_{n}= \pm 1$.

During the summer of 1958 , Salem drew my attention to the problem stated in the first paragraph. It turns out that an affirmative answer can be given by a construction which uses nothing more sophisticated than the parallelogram law

$$
|\alpha+\beta|^{2}+|\alpha-\beta|^{2}=2|\alpha|^{2}+2|\beta|^{2} \text {. }
$$

After I found this construction I learned that the problem had been solved earlier, by essentially the same method, in the 1951 Master's Thesis of H. S. Shapiro [3]. Since the result is needed in Part II of this paper, I am publishing the proof here, with Shapiro's consent. As in the Hardy-Littlewood example, the polynomials (1.1) may actually be taken as the partial sums of a fixed series $\sum_{1}^{\infty} \epsilon_{n} e^{i n \theta}$ :

Theorem I. There exists a sequence $\left\{\epsilon_{n}\right\}(n=1,2,3, \cdots)$, with $\epsilon_{n}=1$ or -1 , such that

$$
\left|\sum_{n=1}^{N} \epsilon_{n} e^{i n \theta}\right|<5 N^{1 / 2} \quad(0 \leqq \theta<2 \pi ; N=1,2,3, \cdots) .
$$

Received by the editors March 26, 1959.

1 The author is a Research Fellow of the Alfred P. Sloan Foundation. This work was supported in part by Air Force Contract SAR/AF 49(638)153. 
Proof. Set $P_{0}(x)=Q_{0}(x)=x$, and define polynomials $P_{k}$ and $Q_{k}$ inductively by

$$
\left\{\begin{array}{l}
P_{k+1}(x)=P_{k}(x)+x^{2^{k}} Q_{k}(x), \\
Q_{k+1}(x)=P_{k}(x)-x^{2^{k}} Q_{k}(x),
\end{array} \quad(k=0,1,2, \cdots) .\right.
$$

Then $P_{k}\left(e^{i \theta}\right)$ is of the form (1.1), with $N=2^{k}$, and $P_{k}$ is a partial sum of $P_{k+1}$. Hence we can define a sequence $\left\{\epsilon_{n}\right\}$ by setting $\epsilon_{n}$ equal to the $n$th coefficient of $P_{k}$, where $2^{k}>n$; this sequence will be shown to have the desired properties.

For $|x|=1,(1.3)$ and (1.5) imply

$$
\begin{aligned}
\left|P_{k+1}(x)\right|^{2}+\left|Q_{k+1}(x)\right|^{2} & =\left|P_{k}(x)+x^{2^{k}} Q_{k}(x)\right|^{2}+\left|P_{k}(x)-x^{2^{k}} Q_{k}(x)\right|^{2} \\
& =2\left|P_{k}(x)\right|^{2}+2\left|Q_{k}(x)\right|^{2},
\end{aligned}
$$

and since $\left|P_{0}(x)\right|^{2}+\left|Q_{0}(x)\right|^{2}=2$, we conclude that

$$
\left|P_{k}\left(e^{i \theta}\right)\right|^{2}+\left|Q_{k}\left(e^{i \theta}\right)\right|^{2}=2^{k+1} \text {. }
$$

Hence

$$
\left|P_{k}\left(e^{i \theta}\right)\right| \leqq 2^{1 / 2} \cdot 2^{k / 2},
$$

which proves (1.4) for $N=2^{k}$.

If now $s_{n}\left(P_{k}\right)$ and $s_{n}\left(Q_{k}\right)$ denote the $n$th partial sums of $P_{k}$ and $Q_{k}$ respectively, where $1 \leqq n \leqq 2^{k}$, then

$$
\left.\mid \begin{array}{l}
s_{n}\left(P_{k}\right)\left(e^{i \theta}\right) \mid \\
s_{n}\left(Q_{k}\right)\left(e^{i \theta}\right) \mid
\end{array}\right\} \leqq\left(2+2^{1 / 2}\right) 2^{k / 2} \quad(k=0,1,2, \cdots) .
$$

This is obviously true if $k=0$. Suppose (1.8) holds for some $k$, and consider $s_{n}\left(P_{k+1}\right)$ and $s_{n}\left(Q_{k+1}\right)$, with $1 \leqq n \leqq 2^{k+1}$. If $n \leqq 2^{k}$, (1.5) shows that

$$
\left|s_{n}\left(P_{k+1}\right)\right|=\left|s_{n}\left(Q_{k+1}\right)\right|=\left|s_{n}\left(P_{k}\right)\right|<\left(2+2^{1 / 2}\right) 2^{(k+1) / 2} .
$$

If $2^{k}<n \leqq 2^{k+1},(1.5)$ and (1.7) show that

$$
\begin{aligned}
\left|s_{n}\left(P_{k+1}\right)\right| & \leqq\left|P_{k}\right|+\left|s_{n-2}^{k}\left(Q_{k}\right)\right| \\
& \leqq 2^{(k+1) / 2}+\left(2+2^{1 / 2}\right) 2^{k / 2}=\left(2+2^{1 / 2}\right) 2^{(k+1) / 2} .
\end{aligned}
$$

The same estimate holds for $\left|s_{n}\left(Q_{k+1}\right)\right|$, and (1.8) is proved by induction.

To complete the proof of (1.4), suppose $2^{k-1} \leqq N \leqq 2^{k}$. By (1.8), we have

$$
\left|s_{N}\left(P_{k}\right)\left(e^{i \theta}\right)\right| \leqq\left(2+2^{1 / 2}\right) 2^{k / 2} \leqq 2\left(1+2^{1 / 2}\right) N^{1 / 2}<5 N^{1 / 2} .
$$

II. Transformations of Fourier coefficients. In this section, $p$ and 
$q$ will always denote conjugate exponents, i.e., $1 / p+1 / q=1$. For $1 \leqq p<\infty, L^{p}$ denotes the usual Lebesgue space of complex functions on the unit circle, normed by

$$
\|f\|_{p}=\left\{\frac{1}{2 \pi} \int_{-\pi}^{\pi}\left|f\left(e^{i \theta}\right)\right|^{p} d \theta\right\}^{1 / p}
$$

$L^{\infty}$ is the space of all essentially bounded measurable functions on the circle. The Fourier coefficients of any $f \in L^{1}$ will be denoted by

$$
\hat{f}(n)=\frac{1}{2 \pi} \int_{-\pi}^{\pi} f\left(e^{i \theta}\right) e^{-i n \theta} d \theta \quad(n=0, \pm 1, \pm 2, \cdots) .
$$

If $F$ is a complex function defined in the plane, we say that $F$ maps $A$ into $B$, where $A$ and $B$ are function spaces on the circle, if to every $f \in A$ there corresponds a $g \in B$ (we shall write: $g=F \circ f$ ) such that $\hat{g}=F(\hat{f})$. In other words, it is required that the series $\sum F\left(c_{n}\right) e^{i n \theta}$ should be the Fourier series of a function in $B$ whenever $\sum c_{n} e^{i n \theta}$ is the Fourier series of a function in $A$.

The functions $F$ which map $L^{1}$ into $L^{1}$ have recently been determined [1]; they are precisely those which are real-analytic near the origin (i.e., in some neighborhood of the origin); of course we must also have $F(0)=0$. For the other Lebesgue spaces, the situation is quite different. We first state some sufficient conditions:

Theorem II. Suppose $1<p \leqq 2$, and suppose there is a constant $A$ such that $|F(z)| \leqq A|z|^{q / 2}$ near the origin. Then $F$ maps $L^{p}$ into $L^{2}$.

Proof. If $f \in L^{p}$, the Hausdorff-Young theorem [4, p. 190] shows that $\sum|\hat{f}(n)|^{a}<\infty$, so that $\sum|F(\hat{f}(n))|^{2}<\infty$.

Theorem III. Suppose $1 \leqq p \leqq 2$. If $|F(z)| \leqq A|z|^{2 / p}$ near the origin, then $F$ maps $L^{q}$ into $L^{q}$.

Proof. If $f \in L^{q}$, then $\sum|\hat{f}(n)|^{2}<\infty$, so that $\sum|F(\hat{f}(n))|^{p}<\infty$, and the Hausdorff-Young theorem implies that $F \circ f \in L^{q}$.

REMARKs. 1. For $q=2$, this condition is necessary as well as sufficient.

2. For $q=\infty$, the hypothesis of Theorem III is: $|F(z)| \leqq A|z|^{2}$. It follows that $F$ maps $L^{\infty}$ (even $L^{2}$ ) into the class of functions which are sums of absolutely convergent trigonometric series.

3 . If $F$ is of the form

$$
F(z)=a_{1} z+a_{2} \bar{z}+|z|^{2 / p} b(z),
$$

where $b$ is a function which is bounded near the origin, then $F$ also maps $L^{q}$ into $L^{q}$. Note that no smoothness conditions are imposed on 
$b$ (not even measurability is needed), in strong contrast to the results in $[1]$.

I do not know whether (2.3) holds whenever $F$ maps $L^{q}$ into $L^{q}$. However, if we restrict ourselves to even functions $F$, Theorem I can be used to show that Theorem III states a condition which is necessary as well as sufficient. In fact, the following stronger assertion holds:

Theorem IV. Suppose $1 \leqq p<\infty, F$ is an even function, and $|z|^{-2 / p}|F(z)|$ is not bounded near the origin. Then there is a continuous function $f$ on the circle to which corresponds no $g \in L^{q}$ with $\hat{g}=F(\hat{f})$.

In other words, $F$ does not map the space of all continuous functions into $L^{q}$, hence it does not map $L^{q}$ into $L^{q}$.

Proof. The hypothesis implies the existence of numbers $z_{m} \neq 0$ $(m=1,2,3, \cdots)$, such that $m^{2} z_{m} \rightarrow 0$ and $\left|F\left(z_{m}\right)\right|>m^{5}\left|z_{m}\right|^{2 / p}$. Define $N_{m}=\left[m^{-4} z_{m}^{-2}\right]$. These choices produce the relations

$$
\sum_{m=1}^{\infty}\left|z_{m}\right| N_{m}^{1 / 2}<\infty
$$

and

$$
\left|F\left(z_{m}\right)\right| N_{m}^{1 / p} \rightarrow \infty \text { as } m \rightarrow \infty .
$$

Now choose integers $n_{m}$ so that

$$
n_{m}+N_{m}<n_{m+1}-N_{m+1}
$$

and define

$$
T_{m}\left(e^{i \theta}\right)=z_{m} e^{i n_{m} \theta}\left(\epsilon_{1} e^{i \theta}+\cdots+\epsilon_{N_{m}} e^{i N_{m} \theta}\right),
$$

where $\left\{\boldsymbol{\epsilon}_{n}\right\}$ is the sequence of Theorem I. The series

$$
f\left(e^{i \theta}\right)=\sum_{m=1}^{\infty} T_{m}\left(e^{i \theta}\right)
$$

converges uniformly, by (2.4) and Theorem I, so that $f$ is contınuous.

Define the kernels $K_{m}$ by

$$
K_{m}\left(e^{i \theta}\right)=e^{i\left(n_{m}+N_{m}\right) \theta} \sum_{n=-2 N_{m}}^{2 N_{m}} \min \left(1,2-\frac{|n|}{N_{m}}\right) e^{i n \theta} .
$$

Suppose there is a function $g \in L^{q}$ such that $\hat{g}=F(\hat{f})$, i.e., $g=F \circ f$. Our choice of $\left\{n_{m}\right\}$ implies that $g * K_{m}=F \circ T_{m}$, where

$$
\left(g * K_{m}\right)\left(e^{i \theta}\right)=\frac{1}{2 \pi} \int_{-\pi}^{\pi} g\left(e^{i(\theta-\phi)}\right) K_{m}\left(e^{i \phi}\right) d \phi .
$$


Since $\left\|K_{m}\right\|_{1}<3$, we see that

$$
\left\|F \circ T_{m}\right\|_{q}<3\|g\|_{q} \quad(m=1,2,3, \cdots) .
$$

On the other hand, the assumption that $F\left(-z_{m}\right)=F\left(z_{m}\right)$ shows that

$$
\left(F \circ T_{m}\right)\left(e^{i \theta}\right)=F\left(z_{m}\right) e^{i n_{m} \theta}\left(e^{i \theta}+\cdots+e^{i N_{m} \theta}\right),
$$

so that

$$
\left|\left(F \circ T_{m}\right)\left(e^{i \theta}\right)\right|=\left|F\left(z_{m}\right)\right| \cdot\left|\frac{\sin \left(N_{m} \theta / 2\right)}{\sin (\theta / 2)}\right| .
$$

An easy computation now yields

$$
\left\|F \circ T_{m}\right\|_{q}>C_{q}\left|F\left(z_{m}\right)\right| N_{m}^{1 / p},
$$

where $C_{q}$ is a positive constant, depending only on $q$. By (2.5), (2.14) implies that $\left\|F \circ T_{m}\right\|_{q} \rightarrow \infty$ as $m \rightarrow \infty$, and this contradicts (2.11).

The theorem follows.

\section{REFERENCES}

1. H. Helson, P. J. Kahane, Y. Katznelson and W. Rudin, The functions which operate on Fourier transforms, Acta Math. vol. 102 (1959) pp. 135-157.

2. Raphael Salem and Antoni Zygmund, Some properties of trigonometrical series whose terms have random signs, Acta Math. vol. 91 (1954) pp. 245-301.

3. H. S. Shapiro, Extremal problems for polynomials and power series, Thesis for S. M. Degree, Massachusetts Institute of Technology, 1951.

4. Antoni Zygmund, Trigonometrical series, Warsaw, 1935.

YALE University 